\title{
GERAdOR DE INDUÇ̃̃o EXCITAdo POR CONVERSOR DE TENSÃo PWM BIDIRECIONAL PARA APLICAÇÕEs RURAis
}

\author{
Enes G. Marra \\ Escola de Engenharia Elétrica - EEE \\ Universidade Federal de Goiás - UFG \\ Goiânia-GO, Brasil \\ e-mail: enes@dsce.fee.unicamp.br
}

\author{
José Antenor Pomilio \\ Faculdade de Engenharia Elétrica e de Computação - FEEC \\ Universidade Estadual de Campinas - UNICAMP \\ C. P. 6101 - CEP: 13081-970, Campinas-SP, Brasil \\ e-mail: antenor@dsce.fee.unicamp.br
}

Resumo - Uma proposta de utilização do gerador de indução auto-excitado com rotor tipo gaiola é apresentada neste artigo. Um conversor PWM bidirecional alimentado em tensão é conectado aos terminais do gerador e mantém a frequiência e a tensão reguladas na carga CA. Apresentam-se resultados de simulação obtidos a partir do modelo que utiliza três eixos $(\alpha \beta \gamma)$ estacionários de referência, empregando-se o programa PSpice ${ }^{\circledR}$. São também apresentados resultados experimentais que descrevem o comportamento do sistema fornecendo energia para diferentes tipos de cargas. $O$ sistema proposto tem por objetivo ser empregado em localidades rurais como uma fonte de tensão senoidal trifásica regulada e frequiência constante. Considera-se que o gerador é acionado por uma turbina hidráulica de pequeno porte sem sistema de regulação mecânico de velocidade, o que resulta em um sistema com baixo custo relativo de instalação e manutenção.

Abstract-This paper concerns an application of a three-phase cage induction machine as self-excited generator. A voltage source PWM bi-directional converter is connected with the generator leads so that frequency and voltage at the $\mathrm{AC}$ load are regulated by the action of this converter. Simulation results were obtained from the $\alpha \beta \gamma$ stationary reference frame model of the induction machine using the PSpice ${ }^{(1)}$ program. The experimental results report the system behavior when feeding different sorts of resistive and inductive loads. The proposed system is intended to be applied in rural sites as a source of regulated three-phase voltage with constant frequency. The generator is supposed to be driven by a low-head hydraulic turbine with an unregulated shaft, which leads to a system with relative low-cost.

\section{INTRODUÇÃO}

A máquina de indução opera como gerador sempre que é mantida excitada e a velocidade do rotor é superior à síncrona $[1,2]$. As principais vantagens da máquina de indução com rotor tipo gaiola operando como gerador são: a robustez e a simplicidade construtiva; capacidade de auto-proteção e baixos níveis de correntes de curto-circuito; necessidade de manutenção reduzida; maior densidade de potência (W/kg) em relação a outros tipos de máquinas; aplicação extremamente difundida, propiciando grande disponibilidade de mercado e baixo custo relativo; e ainda a capacidade de ope- rar como gerador, mesmo quando acionada em velocidade variável.

Apesar destas vantagens, a utilização da máquina de indução apresenta restrições determinantes que deses-timulam o seu emprego como gerador. Entre estas desvantagens destacam-se: regulação de tensão e de freqüência insatisfatórias; necessidade de compensação externa de potência reativa para manutenção da excitação da máquina; variação da freqüência da tensão gerada quando há variação de carga ativa ou reativa alimentada.

O emprego de conversores eletrônicos de potência, tanto para a excitação do gerador, quanto para o processamento da potência gerada tem sido investigado desde as primeiras propostas apresentadas por Arrilaga e Watson [3] até o presente $[4,5]$. O objetivo do sistema proposto neste trabalho é utilizar o gerador de indução movido por turbina hidráulica com velocidade não regulada, para geração de energia elétrica com tensões trifásicas balanceadas e com frequiência constante.

\section{ESTRUTURA DO SISTEMA}

A estrutura do sistema de geração proposto compreende um gerador de indução (GI) auto-excitado por um banco trifásico de capacitores $\left(\mathrm{C}_{\mathrm{CA}}\right)$, e um conversor PWM bidirecional alimentado em tensão (VS-PWM), o qual é conectado aos terminais do gerador através de um filtro passivo de segunda ordem $\left(\mathrm{L}_{\mathrm{C}} \mathrm{C}_{\mathrm{CA}}\right)$, conforme apresentado na figura 1 .

O conversor VS-PWM atua no sistema de forma a manter boa regulação de tensão e frequiência constante nos terminais do gerador.

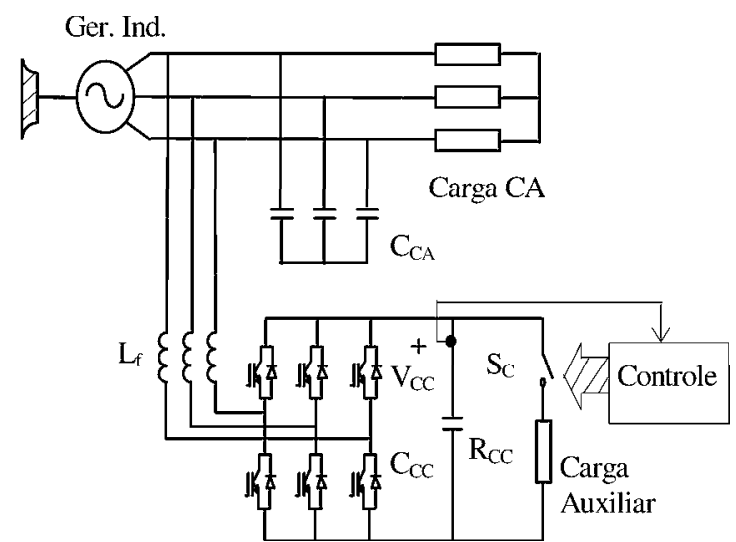

Figura 1. Estrutura geral do sistema de geração com controle de tensão pela carga $\mathrm{CC}$. 
Um capacitor $\left(\mathrm{C}_{\mathrm{CC}}\right)$, presente no lado $\mathrm{CC}$ do conversor, atua como fonte de tensão contínua, dispensando o emprego de outras fontes externas.

O conversor é controlado em malha aberta por modulação PWM com a técnica de modulação vetorial (space vector modulation). A freqüência fundamental da tensão de saída do conversor é fixada em $60 \mathrm{~Hz}$, criando-se assim um barramento de freqüência constante para o GI. Desta maneira, o gerador opera seguindo a curva característica de conjugado para a frequência de $60 \mathrm{~Hz}$, conforme indicado pela figura 2 .

Pretende-se que o gerador seja acionado por uma turbina hidráulica sem regulador mecânico de velocidade, resultando em velocidade variável para o rotor, numa faixa de variação que pode ser considerada estreita se comparada ao caso de acionamento por turbina eólica.

Este sistema requer uma coordenação entre a curva de conjugado da turbina hidráulica e a família de curvas de conjugado da máquina de indução, a fím de que o valor máximo possível da potência desenvolvida pela turbina corresponda a um ponto no qual o escorregamento de operação do gerador seja inferior ao escorregamento nominal. $O$ ponto "A" da figura 2 apresenta um possível ponto de operação do sistema para a curva de conjugado de uma dada turbina hidráulica não regulada.

Considerando-se que a tensão CA do conversor apresenta frequiência constante, a potência elétrica gerada é determinada pela potência mecânica fornecida ao sistema. A potência mecânica, por sua vez, é determinada pela associação da curva característica de conjugado por velocidade do GI com a curva característica de conjugado por velocidade da turbina.

Assim sendo, a potência elétrica gerada é independente da carga elétrica alimentada pelo gerador. Toda potência elétrica gerada deve ser consumida, de modo a manter o balanço de potência do sistema. Se a carga CA não é suficiente para consumir toda a potência, o excedente é armazenado no capacitor $\mathrm{C}_{\mathrm{CC}}$.

O sistema deve prover alguma estratégia para consumir o excesso de potência, a fim de manter-se a tensão CC do conversor $\left(\mathrm{V}_{\mathrm{CC}}\right)$ constante. Isto sendo feito, a amplitude da

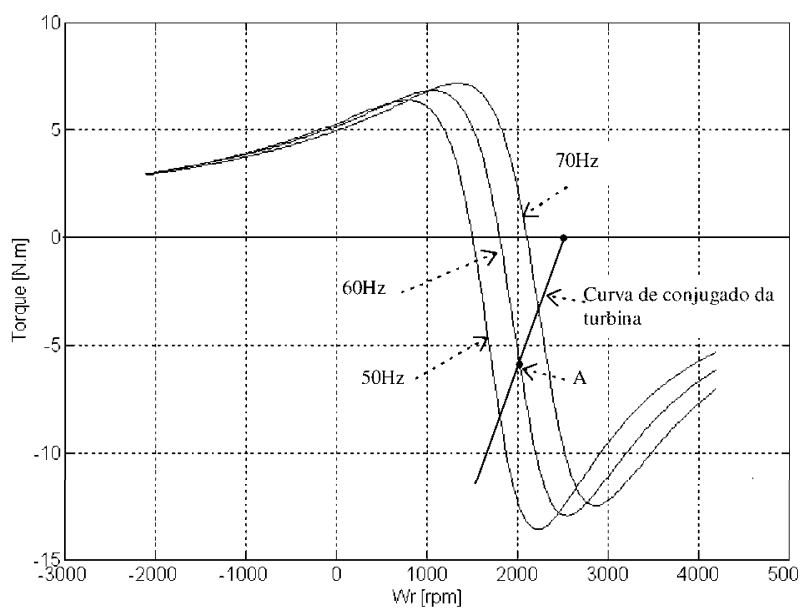

Figura 2. Família de curvas de conjugado por velocidade para a máquina de indução. tensão de linha no lado CA do conversor é também mantida constante, pois o capacitor $C_{C C}$ opera como fonte de tensão contínua para o conversor. Desta forma, uma boa regulação da tensão terminal é naturalmente obtida, uma vez que a diferença entre a tensão terminal do GI e a tensão terminal CA do conversor VS-PWM é somente a tensão sobre a indutância série $\mathrm{L}_{\mathrm{f}}$.

A partida do sistema ocorre com a aceleração do gerador pela máquina primária, que passa a produzir tensão terminal proporcional à velocidade e ao fluxo residual. A interação entre a esta tensão e o banco trifásico de capacitores de excitação do gerador $\left(\mathrm{C}_{\mathrm{CA}}\right)$ dá início ao processo de autoexcitação do gerador. $\mathrm{O}$ capacitor $\mathrm{C}_{C \mathrm{C}}$ é carregado com energia provida pelo próprio GI durante o período de autoexcitação. Ao final deste período, a tensão terminal CA do gerador e a tensão $V_{c c}$ estão estabelecidas.

Os circuitos de controle do conversor VS-PWM são alimentados pelo capacitor $\mathrm{C}_{\mathrm{CC}}$, através de um conversor $\mathrm{CC}$ CC tipo forward. O inversor é então habilitado, e passa a operar com frequiência constante. A partir deste ponto, a freqüência síncrona passa a ser ditada pelo inversor.

Além da potência inicialmente requerida para o carregamento do capacitor $\mathrm{C}_{\mathrm{CC}}$ durante a partida do sistema e para alimentação dos circuitos de controle do conversor, o gerador de indução supre também a potência relativa às perdas no sistema durante a operação.

No momento da habilitação do conversor, há o aparecimento de uma corrente transitória, provocada pela diferença instantânea entre a tensão terminal do gerador e a tensão terminal do inversor. Esta corrente transitória é limitada inicialmente pelas indutâncias série $\left(\mathrm{L}_{\mathrm{f}}\right)$ e não chega a atingir valores muito superiores à corrente nominal da máquina.

Baixo valor de corrente de curto-circuito é uma das características naturais do gerador de indução. Esta característica impede o aparecimento de corrente transitória sustentada durante muitos ciclos, no instante do início de operação do conversor. Quando a corrente transitória manifesta uma tendência de crescimento elevado, o GI perde momentaneamente a excitação, e é imediatamente reexcitado pela ação do conversor, tendo $\mathrm{C}_{\mathrm{CC}}$ como fonte de tensão. Todo este processo normalmente dura em torno de quatro ciclos de $60 \mathrm{~Hz}$.

Os indutores $\mathrm{L}_{\mathrm{f}}$ em associação com os capacitores $\mathrm{C}_{\mathrm{CA}}$ atenuam as componentes de alta frequiência da tensão $\mathrm{CA}$ do conversor, tornando senoidal a tensão terminal do GI.

$\mathrm{O}$ primeiro passo na determinação dos componentes do filtro passivo passa-baixas $\left(\mathrm{L}_{\mathrm{C}} \mathrm{C}_{\mathrm{CA}}\right)$ é a escolha do valor da capacitância $\mathrm{C}_{\mathrm{CA}}$, a qual depende unicamente da demanda de potência reativa necessária para a excitação do gerador de indução durante a partida do sistema. A capacitância $\mathrm{C}_{\mathrm{CA}}$ pode ser obtida a partir da curva de excitação da máquina de indução.

Uma vez especificada $\mathrm{C}_{\mathrm{CA}}$, a indutância $\mathrm{L}_{\mathrm{f}}$ é determinada segundo (1).

$$
\mathrm{L}_{\mathrm{f}}=\frac{1}{\left(2 \pi \mathrm{f}_{\mathrm{C}}\right)^{2} \mathrm{C}_{\mathrm{CA}}}
$$


A freqüência de corte $\left(f_{c}\right)$ deve ser definida acima da freqüência fundamental da tensão terminal do GI e pelo menos uma década abaixo da frequiência de chaveamento do conversor VS-PWM.

O sistema proposto gera energia elétrica com qualidade suficiente para alimentar cargas sensíveis, como, por exemplo, cargas controladas por microprocessadores. Além disto, a estratégia de controle proposta evita o emprego de controladores de alta complexidade, e também o ônus do investimento em reguladores mecânicos de velocidade para a turbina hidráulica.

O baixo custo relativo, a robustez e a boa qualidade da energia gerada são as principais vantagens desta aplicação proposta para o GI em operação isolada. Visando manter estas características, admite-se inclusive o emprego da energia excedente em cargas dissipativas como parte do processo de controle da tensão $\mathrm{V}_{\mathrm{CC}}$ do conversor [6].

\section{A. Controle de Tensão pela Carga CC}

$\mathrm{O}$ excedente de energia proveniente da diferença entre a potência gerada e a potência consumida pela carga $\mathrm{CA}$ pode ser empregado em cargas $\mathrm{CC}$ como estratégia para regulação da tensão $V_{c c}$. A potência consumida por tais cargas pode ser utilizada em aplicações tais como um sistema de co-geração de energia, no carregamento de um grupo de baterias, em sistemas de aquecimento, ou na associação de algumas destas aplicações.

Neste caso, o controle da tensão $V_{C C}$ é realizado através um sistema resistivo de aquecimento $\left(\mathrm{R}_{\mathrm{CC}}\right)$, conforme apresentado na figura 1 . O controle da chave $S_{C}$ é obtido através da comparação por histerese da tensão $\mathrm{V}_{\mathrm{CC}}$, de forma a mantê-la variando em torno de um valor referência [6].

\section{B. Controle de Tensão pela Carga $C A$}

Outra alternativa de controle para a tensão $V_{C C}$ é através de uma carga CA controlável, cuja potência é variada de forma que a potência excedente é por ela consumida.

$O$ controle da tensão $V_{C C}$ pela carga $C A$ elimina a necessidade do trânsito da potência excedente através do conversor VS-PWM, reduzindo assim sua potência nominal em relação ao sistema de controle de tensão pela carga $\mathrm{CC}$.

A figura 3 apresenta a estrutura do sistema no qual é utilizado o controle de tensão pela carga CA. A carga CA controlável pode consistir, por exemplo, de um sistema de aquecimento, composto por um banco trifásico de resistores conectados em delta e controlados por SCRs em anti-paralelo (conforme a figura 3), ou ainda de um sistema de bombeamento de água para armazenamento em reservatórios ou para irrigação.

$\mathrm{O}$ sinal de tensão $\mathrm{V}_{\mathrm{cc}}$ é empregado como sinal de realimentação para o controlador PI, o qual gera o sinal de referência $\left(\mathrm{S}_{\mathrm{REF}}\right)$ para o sistema de comando dos SCRs.

$O$ valor da tensão $V_{c c}$ é um parâmetro capaz de oferecer um rápido diagnóstico do balanço de potência no sistema. Se o nível da tensão $V_{C C}$ sofre elevação, isto indica um excedente de potência. Por outro lado, a queda da tensão $V_{C C}$ revela que a potência consumida pela carga CA é superior à potência gerada naquele momento.

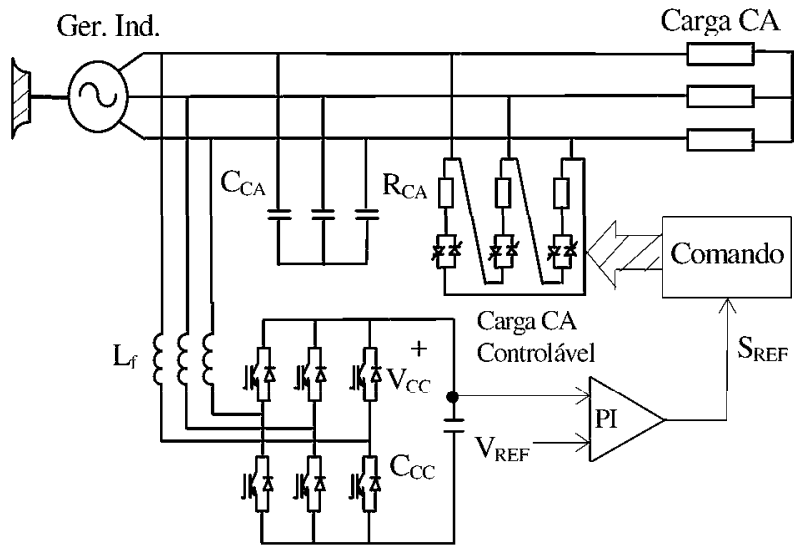

Figura 3. Estrutura geral do sistema de geração com controle de tensão pela carga CA.

\section{MODELO DA MÁQUINA DE INDUÇÃO}

Neste estudo, a máquina é representada por um modelo trifásico a três condutores, segundo o qual as grandezas e os parâmetros do rotor são referidos a um sistema de três eixos estacionários em relação ao estator, denominados eixos $\alpha \beta \gamma$. O desenvolvimento completo deste modelo é apresentado nas referências $[7,8]$.

As principais vantagens do modelo $\alpha \beta \gamma$ são: a utilização de um circuito equivalente com mesmo número de terminais da máquina; a possibilidade de simulação dinâmica da máquina utilizando-se programas que empregam representação circuital; as grandezas do estator não são afetadas pela transformação $\alpha \beta \gamma$; o modelo apresenta boa precisão e robustez matemática. Este modelo permite também inclusão de desequilíbrios elétricos, desde que o circuito elétrico da máquina seja trifásico a três condutores.

A determinação do modelo da máquina de indução, referida ao sistema de eixos estacionários $\alpha \beta \gamma$ é desenvolvido a partir das seguintes considerações: as grandezas do rotor são referidas ao estator pela utilização da relação de espiras entre rotor e estator; não há saturação magnética da máquina, a força magnetomotriz (FMM) não apresenta componentes harmônicas espaciais, as perdas magnéticas e mecânicas são desprezíveis, e a máquina é trifásica a três condutores.

As posições espaciais dos eixos $\alpha \beta \gamma$ da referência estacionária são, respectivamente, coincidentes com as posições espaciais dos eixos magnéticos dos enrolamentos estatóricos das fases abc da máquina para cada par de pólos.

A transformação do sistema de referência é feita de acordo $\operatorname{com}(2)$.

$$
\left[\mathbf{f}_{\alpha \beta \gamma}\right]=\left[\mathrm{K}_{\alpha}^{\mathrm{a}}\right] .\left[\mathrm{f}_{\mathrm{abc}}\right]
$$

$\left[f_{\alpha \beta \gamma}\right]$ e $\left[f_{a b c}\right]$ são grandezas referidas ao sistema de eixos $\alpha \beta \gamma$ e ao sistema de eixos abc respectivamente.

$\left[\mathrm{K}_{\alpha}^{\mathrm{a}}\right]$ é a matriz de transformação da referência abc para a referência $\alpha \beta \gamma$, a qual pode ser decomposta em uma matriz de transformação para o estator $\left[\mathrm{K}_{\gamma \mathrm{s}}^{\mathrm{a}}\right.$ ], e uma outra matriz 
de transformação para o rotor $\left[\mathrm{K}_{\alpha \mathrm{r}}^{\mathrm{a}}\right]$, conforme apresentado por (3) e (4).

$$
\begin{gathered}
{\left[\mathrm{K}_{\alpha \mathrm{s}}^{\mathrm{a}}\right]=\operatorname{diag}\left[\begin{array}{lll}
1 & 1 & 1
\end{array}\right]} \\
{\left[\mathrm{K}_{\alpha \mathrm{r}}^{\mathrm{a}}\right]=\left[\begin{array}{ccc}
\cos \theta_{\mathrm{r}}+\frac{1}{2} & \cos \left(\theta_{\mathrm{r}}+\frac{2}{3}\right)+\frac{1}{2} & \cos \left(\theta_{\mathrm{r}}-\frac{2}{3}\right)+\frac{1}{2} \\
\cos \left(\theta_{\mathrm{r}}-\frac{2}{3}\right)^{+\frac{1}{2}} & \cos \theta_{\mathrm{r}}+\frac{1}{2} & \cos \left(\theta_{\mathrm{r}}+\frac{2}{3}\right)+\frac{1}{2} \\
\cos \left(\theta_{\mathrm{r}}+\frac{2}{3}\right)+\frac{1}{2} & \cos \left(\theta_{\mathrm{r}}-\frac{2}{3}\right)^{+\frac{1}{2}} & \cos \theta_{\mathrm{r}}+\frac{1}{2}
\end{array}\right]}
\end{gathered}
$$

$\theta_{\mathrm{r}}$ é a posição angular elétrica do rotor em função do tempo, dada em radianos.

As grandezas do estator não são afetadas pela transformação de sistema de referência. Assim, a notação das grandezas do estator é mantida com a mesma designação do sistema de referência ahc.

A aplicação de (2) a (4) para determinação do modelo dinâmico da máquina de indução resulta em:

$$
\begin{gathered}
{\left[\mathrm{v}_{\mathrm{abcs}}\right]=\mathrm{r}_{\mathrm{s}}\left[\mathrm{i}_{\mathrm{abcs}}\right]+\frac{\mathrm{d}\left[\lambda_{\mathrm{abcs}}\right]}{\mathrm{dt}}} \\
0=\mathrm{r}_{\mathrm{r}}\left[\mathrm{i}_{\alpha \beta \gamma \mathrm{r}}\right]+\frac{\mathrm{d}\left[\lambda_{\alpha \beta \gamma \mathrm{r}}\right\rfloor}{\mathrm{dt}}+\frac{\omega_{\mathrm{r}}}{\sqrt{3}}\left[\lambda_{\alpha \mathrm{x}}\right] \\
\left.\left[\lambda_{\mathrm{abcs}}\right]=\left(\mathrm{L}_{\mathrm{ls}}+\mathrm{M}\right)\left[\mathrm{i}_{\mathrm{abcs}}\right]+\mathrm{M} \mid \mathrm{i}_{\alpha \beta \gamma \mathrm{r}}\right\rfloor \\
{\left[\lambda_{\alpha \beta \gamma \mathrm{r}}\right\rfloor=\left(\mathrm{L}_{\mathrm{lr}}+\mathrm{M}\right)\left[\mathrm{i}_{\alpha \beta \gamma \mathrm{r}}\right\rfloor+\mathrm{M}\left[\mathrm{i}_{\mathrm{abcs}}\right]} \\
{\left[\lambda_{\alpha \mathrm{x}}\right]=\left[\begin{array}{c}
\left(\lambda_{\beta \mathrm{r}}-\lambda_{\gamma \mathrm{r}}\right) \\
\left(\lambda_{\gamma \mathrm{r}}-\lambda_{\alpha \mathrm{r}}\right) \\
\left(\lambda_{\alpha \mathrm{r}}-\lambda_{\beta \mathrm{r}}\right)
\end{array}\right]} \\
\mathrm{T}_{\mathrm{e}}=\sqrt{3}\left(\frac{\mathrm{P}}{2}\right) \mathrm{M}\left(\mathrm{i}_{\mathrm{as}} \mathrm{i}_{\gamma \mathrm{r}}-\mathrm{i}_{\mathrm{cs}} \mathrm{i}_{\alpha \mathrm{r}}\right)
\end{gathered}
$$

O subscrito abcs indica as grandezas do estator e o subscrito $\alpha \beta \gamma r$ indica as grandezas do rotor, ambas referidas ao sistema estacionário $\alpha \beta \gamma$ no estator. $\mathrm{L}_{\mathrm{ls}}$ e $\mathrm{L}_{\mathrm{l} r}$ são as respectivas indutâncias de dispersão dos enrolamentos do estator e do rotor, obtidas através do ensaio com rotor bloqueado. $\mathrm{M}$ é a indutância mútua entre os enrolamentos do estator e do rotor, obtida através do ensaio a vazio. $T_{e}$ é o conjugado eletromagnético. $\left[\mathrm{v}_{\mathrm{abcs}}\right]$ e $\left[\mathrm{i}_{\mathrm{abcs}}\right]$ são os vetores das tensões e das correntes de estator, respectivamente. $\left[\mathrm{I}_{\alpha \beta \gamma \mathrm{\gamma}}\right]$ é o vetor das correntes de estator. $\left[\lambda_{\text {abcs }}\right]$ e $\left[\lambda_{\alpha \beta \gamma r}\right]$ são os vetores de fluxo magnético concatenado pelos enrolamentos de estator e de rotor, respectivamente. $r_{s}$ e $r_{r}$ são as respectivas resistências dos enrolamentos do estator e do rotor. $O$ vetor de fluxos $\left[\lambda_{\alpha x}\right]$ resulta da transformação matemática entre o sistema de referência abc para o sistema de referência $\alpha \beta \gamma$. $\omega_{\mathrm{r}}$ é a velocidade angular elétrica do eixo do rotor.

A eq. (11) descreve o comportamento mecânico do sistema.

$$
T_{e}=\left(\frac{2}{p}\right) J \frac{d \omega_{r}}{d t}+\left(\frac{2}{p}\right) B_{m} \omega_{r}+T_{L}
$$

P é o número de pólos da máquina; J é a constante de inércia do rotor; $B_{m}$ é a constante de atrito rotacional; e $T_{L}$ é o conjugado de carga.

O modelo da máquina de indução trifásica referido ao sistema de eixos estacionários $\alpha \beta \gamma$ do estator é ditado pelas eqs. (5) a (11).

A modelagem $\alpha \beta \gamma$ não afeta as grandezas do estator, contrariamente ao que ocorre no modelo $\mathrm{D}^{\mathrm{e}}-\mathrm{Q}^{\mathrm{e}}$ ou $\mathrm{D}^{\mathrm{s}}-\mathrm{Q}^{\mathrm{s}}$ da máquina de indução. Deste modo, qualquer dispositivo pode ser conectado aos terminais de estator do circuito que representa o modelo dinâmico $\alpha \beta \gamma$ da MI, sem que seja necessário aplicar qualquer transformação matemática. Assim, para efeito de simulação, o circuito do conversor VS-PWM pode ser conectado ao estator da MI, sem a necessidade de aplicar a transformação $\alpha \beta \gamma$ ao conversor.

\section{SIMULAÇÃO DO SISTEMA}

O sistema de geração com controle de tensão pela carga CC foi simulado com o programa PSpice ${ }^{\mathbb{1}}$, empregando-se o modelo $\alpha \beta \gamma$ para a máquina de indução.

Considerando-se que a operação do gerador ocorre com frequiência síncrona constante e tensão terminal regulada, o modelo linear da máquina de indução representa o comportamento dinâmico da MI de maneira satisfatória.

Os parâmetros da máquina de indução representada na simulação estão descritos na tabela I. Os valores dos demais componentes apresentados na figura 1 são: $\mathrm{C}_{\mathrm{CA}}=37 \mu \mathrm{F}$, $\mathrm{L}_{\mathrm{f}}=5 \mathrm{mH}$ e $\mathrm{R}_{\mathrm{CC}}=120 \Omega$.

A figura 4 apresenta a ação do controle de tensão pela carga $C C$. Neste caso, $V_{c c}$ é comparado por histerese com um valor de referência e o sinal resultante desta comparação

TABELA I

Parâmetros da máquina de indução em $60 \mathrm{~Hz}$.

\begin{tabular}{ll}
\hline Resistência de estator $\left(\mathrm{r}_{\mathrm{S}}\right)$ & $4,4 \Omega$ \\
\hline Resistência do rotor $\left(\mathrm{r}_{\mathrm{r}}\right)$ & $5,02 \Omega$ \\
\hline Reatância de dispersão do estator $\left(\mathrm{X}_{\mathrm{S}}\right)$ & $5,9 \Omega$ \\
\hline Reatância de dispersão do rotor $\left(\mathrm{X}_{\mathrm{r}}\right)$ & $5,9 \Omega$ \\
\hline Reatância mútua $\left(\mathrm{X}_{\mathrm{m}}\right)(2 \pi .60 . \mathrm{M})$ & $70,53 \Omega$ \\
\hline $\begin{array}{l}\text { Resistência de perdas mecânicas e elétricas } \\
\left(\mathrm{R}_{\mathrm{m}}\right)\end{array}$ & $582 \Omega$ \\
\hline Potência nominal & $1 / 2 \mathrm{cv}$ \\
\hline Número de pólos $(\mathrm{P})$ & 4 \\
\hline Inércia do rotor $(\mathbf{J})$ & $0,0006 \mathrm{~kg} \cdot \mathrm{m}^{2}$ \\
\hline
\end{tabular}

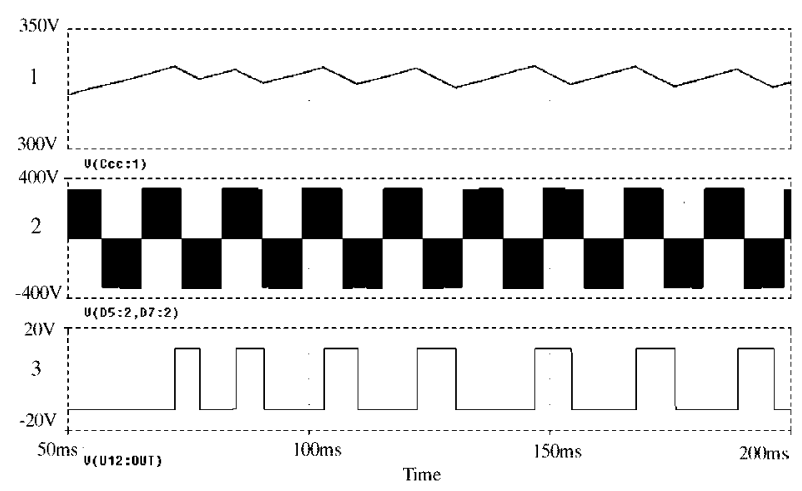

Figura 4. Tensão $V_{C C}$ (1); Tensão de linha do conversor VS-PWM (2); Sinal de comando da chave $\mathrm{S}_{\mathrm{C}}(3)$. 
determina o estado da chave $S_{C}$. A largura da banda de histerese é determinada de forma que a tensão $V_{C C}$ varie dentro de limites aceitáveis.

A simulação da conexão transitória de uma carga com potência aproximada de $50 \%$ da potência nominal do gerador é apresentada na figura 5. Após a partida do sistema entre 0s e $50 \mathrm{~ms}$, um banco de resistências é conectado como carga para o GI e mantido até $150 \mathrm{~ms}$. Durante o período de conexão da carga, a elevação da tensão $V_{C C}$ é lenta e não requer o fechamento da chave $S_{C}$. Após a desconexão da carga, em $150 \mathrm{~ms}$, o capacitor $\mathrm{C}_{\mathrm{CC}}$ passa a acumular maior quantidade de energia, e a ação do chaveamento do resistor $\mathrm{R}_{\mathrm{CC}}$ mantém a tensão $\mathrm{V}_{C C}$ dentro da banda de histerese estabelecida.

\section{RESULTADOS EXPERIMENTAIS}

A implementação experimental do sistema com controle de tensão pela carga CC (figura 1) foi realizada com componentes cujos valores são iguais aos utilizados nas simulações e com uma máquina de indução cujos parâmetros são apresentados na tabela I. Um conversor de frequiência disponível comercialmente no mercado foi empregado como conversor bidirecional VS-PWM.

A figura 6 apresenta as tensões terminais de linha no conversor VS-PWM e no GI. A forma de onda senoidal da tensão do gerador mostra que a ação do filtro $\mathrm{L}_{\mathrm{f}} \mathrm{C}_{\mathrm{CA}}$ é efetiva na atenuação das componentes de alta frequiência presentes na tensão CA do conversor.

As figuras 7 e 8 apresentam as correntes de linha no gerador, no conversor e na carga CA (resistiva), para situações de carga nominal (figura 7) e a vazio (figura 8). Observa-se que a corrente terminal do gerador não é afetada pela potência da carga CA em regime permanente.

Uma comparação entre as figuras 7 e 8 mostra que o aumento da potência da carga CA resulta em diminuição da corrente de linha no conversor VS-PWM, como resultado do balanço entre a potência gerada e a potência consumida.

A corrente de linha do gerador compreende a corrente de excitação da máquina, a corrente da carga CA e a corrente que flui para o conversor VS-PWM. Neste caso, por tratar-se de uma máquina de pequeno porte, a corrente de excitação do gerador tem grandeza equivalente à corrente da carga.

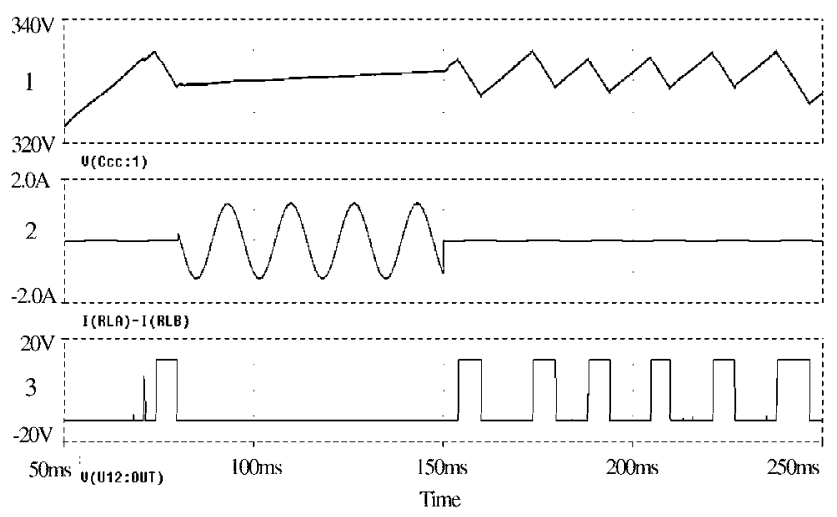

Figura 5. Tensão $V_{C C}$ (1); Corrente de linha na carga $C A$ (2); Sinal de comando da chave $S_{C}(3)$.
As componentes de alta frequiência observadas na corrente de linha do conversor são absorvidas pelos capacitores $\mathrm{C}_{\mathrm{CA}}$, desta forma não há componentes de alta frequiência na corrente do gerador.

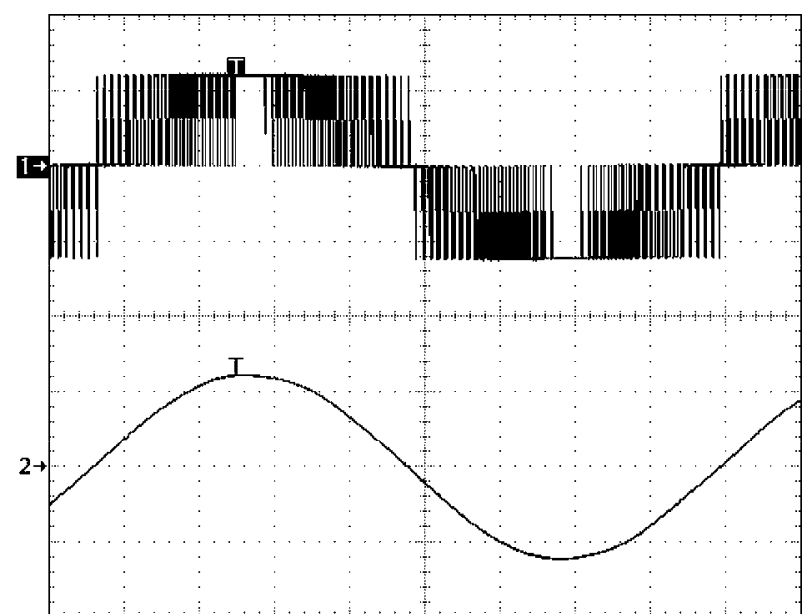

Figura 6. Tensões terminais de linha no conversor VS-PWM (1) e no gerador de indução (2). Vert.: $250 \mathrm{~V} /$ div. Horiz.: $2 \mathrm{~ms} / \mathrm{div}$.

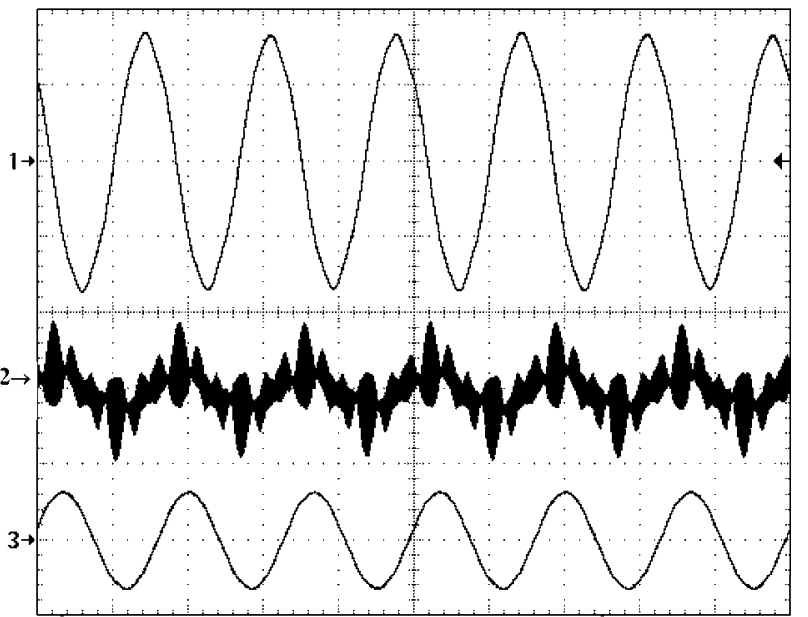

Figura 7. Operação com carga nominal. Correntes de linha no gerador (1), no conversor (2) e na carga CA (3). Vert.: 2A/div. Horiz.: $10 \mathrm{~ms} / \mathrm{div}$.

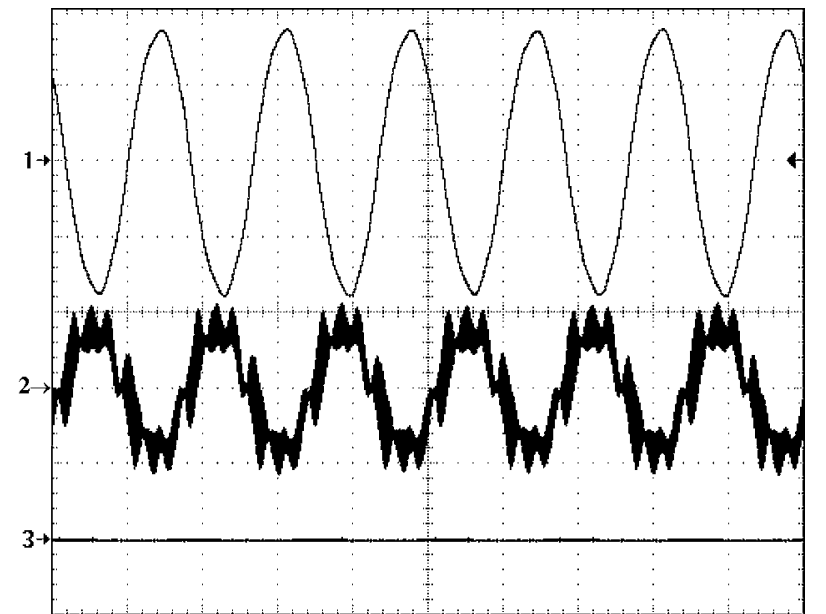

Figura 8. Operação a vazio. Correntes de linha no gerador (1), no conversor (2) e na carga CA (3). Vert.: 2A/div. Horiz.: $10 \mathrm{~ms} /$ div. 
O sistema de geração com controle de tensão pela carga CA (figura 3) foi também implementado experimentalmente.

A figura 9 apresenta uma sequiência de transitórios de carga realizados através da conexão consecutiva de três grupos de cargas resistivas. A carga do gerador variou desde de $0 \%$ até $100 \%$ da sua potência nominal.

A medida que a carga aumenta, a referência para o ângulo de disparo dos SCRs da carga CA ajustável também é incrementada pela ação do controlador PI, resultando em maior ângulo de disparo e menor tempo de condução.

A partida direta de um motor de indução trifásico, cuja potência nominal é de $67 \%$ da potência do gerador de indução, foi realizada com sucesso, empregando-se quaisquer das estratégias de controlc de tensão.

A figura 10 apresenta a tensão $V_{C C}$, a tensão terminal do gerador e a corrente de linha no motor. A tensão terminal do gerador segue um perfil de comportamento semelhante ao da tensão $V_{r r}$.

Durante a partida, grande parte da energia requerida para a aceleração do motor é fornecida pelo capacitor $\mathrm{C}_{\mathrm{cc}}$. Assim, a máxima variação de tensão transitória na partida de motores pode ser tomada como parâmetro para a determinação do valor de $\mathrm{C}_{\mathrm{CC}}$.

$O$ capacitor $C_{C C}$ deve ser elevado o suficiente para evitar oscilações (ripple) na tensão $\mathrm{V}_{\mathrm{CC}}$. Entretanto, constatou-se experimentalmente que quando $\mathrm{C}_{\mathrm{CC}}$ é dimensionado para satisfazer à partida de um motor de indução, cuja potência é significativa em relação à potência nominal do GI, o ripple em $V_{c c}$ é desprezível.

O conversor fornece a corrente de magnetização do motor, já que o banco trifásico de capacitores $\mathrm{C}_{\mathrm{CA}}$ é dimensionado para atender apenas a potência reativa requerida para a excitação do gerador.

O gerador de indução necessita de compensação externa de reativos para manter sua corrente de magnetização. Em sistemas isolados da rede elétrica, a forma mais simples de prover esta compensação é através de um banco trifásico de capacitores. Por este motivo, quando uma carga indutiva é

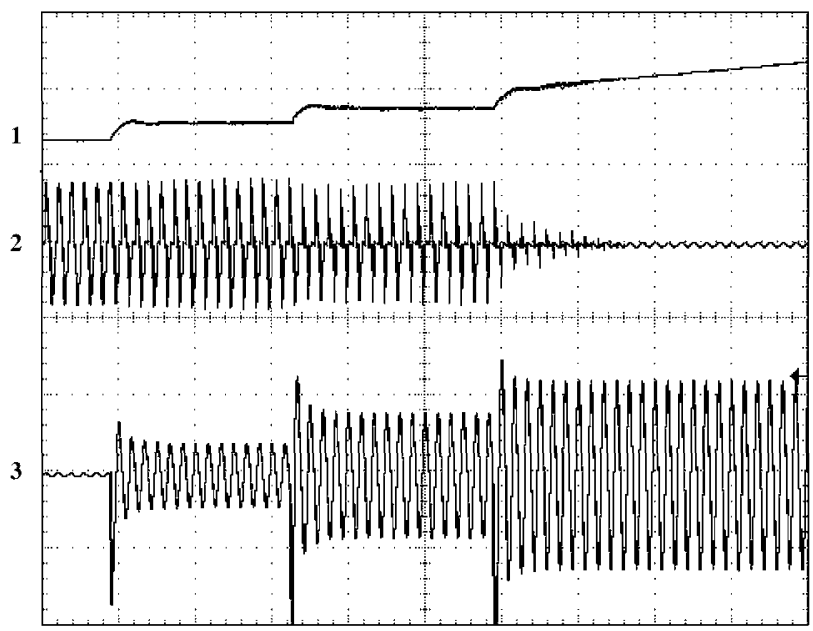

Figura 9. Tensão de referência para o comando dos SCRs (1) (5V/div.); corrente de linha na carga CA controlável (2) (2A/div.), e na carga CA (3) (1A/div.); Horiz.: 100ms/div.

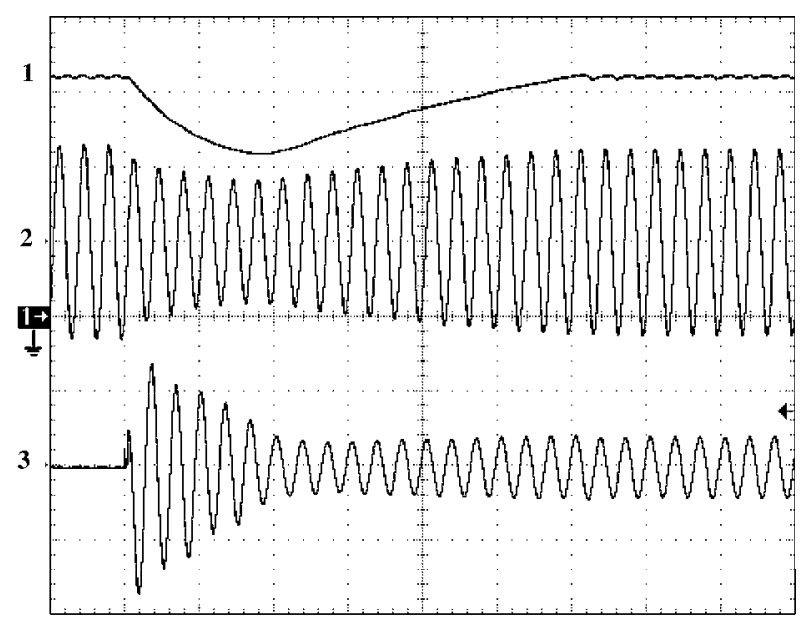

Figura 10. Tensão $\mathrm{V}_{\mathrm{CC}}(100 \mathrm{~V} /$ div. $)$ (1); tensão terminal do gerador (250V/div.) (2) e corrente de linha no motor durante a partida

(5A/div.) (3). Horiz.: 50ms/div.

conectada ao gerador, é necessário aumentar a capacidade de compensação do bando de capacitores CA; caso contrário, o gerador é desmagnetizado, e ocorre o colapso de sua tensão terminal.

No presente sistema, a conexão do conversor VS-PWM ao gerador, aliado à estratégia adotada para o controle da tensão $\mathrm{V}_{\mathrm{CC}}$, mantêm a tensão terminal do gerador, mesmo durante a alimentação de cargas indutivas com potência de 1 pu.

Após a partida do sistema, até mesmo o banco $\mathrm{C}_{\mathrm{CA}}$ poderia ser retirado, sem prejuízo para a magnetização do gerador. Isto só não é realizado devido à necessidade da ação do filtro $\mathrm{L}_{\mathrm{i}} \mathrm{C}_{\mathrm{CA}}$, e porque o banco $\mathrm{C}_{\mathrm{CA}}$ auxilia o conversor a suprir a demanda por reativos.

Considerando-se que o capacitor $\mathrm{C}_{\mathrm{CC}}$ é a fonte de tensão do conversor, que a tensão $V_{C C}$ é praticamente constante e que o conversor VS-PWM permite fluxo bidirecional de potência, a atuação do conversor como compensador de reativos ocorre como uma consequiência natural da forma de operação do sistema.

O sistema apresentou uma satisfatória regulação de tensão de $1,8 \%$ para cargas resistivas e de $2,2 \%$ para cargas indutivas. Os testes realizados mostraram também que o desbalanço da carga CA não afeta a tensão do GI.

O sistema mostrou-se capaz de suprir retificadores trifásicos a diodo. Neste caso, as correntes harmônicas produzidas pelos retificadores dividem-se entre o banco de capacitores $\mathrm{C}_{\mathrm{CA}}$, o circuito de estator do GI, e o conversor VS-PWM, através das indutâncias $\mathrm{L}_{\text {q }}$.

A forma com que as correntes harmônicas distribuem-se entre estes circuitos depende do valor das impedâncias de cada um dos circuitos para uma dada freqüência harmônica.

\section{CONCLUSÃO}

Este trabalho apresentou um sistema no qual geradores de indução auto-excitados tipo gaiola podem alimentar cargas trifásicas resistivas ou indutivas, equilibradas ou desequilibradas, lineares ou não-lineares com boa regulação de tensão e freqüência constante.

A qualidade da energia fornecida, o baixo custo relativo do sistema e a não utilização de reguladores mecânicos de 
velocidade são importantes diferenciais deste trabalho em comparação com outras propostas de emprego dos geradores de indução.

O gerador de indução é o dispositivo conversor de potência do sistema. Enquanto, o conversor VS-PWM atua como compensador de reativos, como regulador de tensão e como regulador de frequiência.

$O$ controle da tensão $V_{C c}$ apresentou-se como uma estratégia bastante eficiente na obtenção de uma boa regulação para a tensão gerada.

A tensão $V_{C c}$ é controlada através do consumo da potência proveniente da diferença entre a potência gerada e a potência consumida pela carga CA. Esta diferença de potência deve ser consumida por cargas auxiliares.

Neste sistema não é necessário o uso de sensores de tensão ou de transformadores de corrente ou de potencial e o único sinal requerido pelo controle é a tensão $\mathrm{V}_{\mathrm{CC}}$, a qual é amostrada através de um divisor resistivo.

A utilização de uma máquina de indução e de um conversor de frequiência disponíveis no mercado é também um dos destaques desta proposta.

$O$ banco de capacitores $\mathrm{C}_{\mathrm{CA}}$ possibilita a auto-excitação do gerador de indução na partida do sistema através do fluxo residual da máquina, sem a necessidade de um dispositivo específico para esta finalidade. Este banco atua ainda como um filtro para as componentes de alta frequiência da tensão CA do conversor, assegurando tensão terminal senoidal para o gerador.

O sistema mostrou-se estável e robusto ao permitir a partida direta de um motor de indução cuja potência é de $67 \%$ da potência do gerador.

\section{AGRADFCIMENTOS}

Os autores agradecem o apoio financeiro da CAPES, da FAPESP e do CNPq e a colaboração do Prof. Rodolfo M. M. Martinez na simulação da máquina e do Sr. Leonardo de Araujo Silva na implementação do comando da carga CA ajustável.

\section{REFERÊNCIAS BIBLIOGRÁFICAS}

[1] D. E. Basset, M. F. Potter, M. F. "Capacitive excitation for induction generators", AIEE Transactions, Vol. 54, p. 540-543, 1935.

[2] C. F. Wagner. "Self-excitation of induction motors", AIEE Transactions, Vol. 58, p. 47-51, Feb. 1939.

[3] J. Arrilaga, D. B. Watson. "Static power conversion from self-excited induction generators", IEE Proc., Vol. 125, No. 8, p. 743, 1978.
[4] M. G. Simões, B. K. Bose, R. J. Spiegel. "Fuzzy logic based intelligent control of a variable speed cage machine wind generation system", Proceedings of PESC'95, p. 389-395, 1995.

[5] C. B. Jacobina, E. R. C. Silva, A. M. N. Lima, R. L. A. Ribeiro. "Induction generator static systems with a reduced number of components", Proceedings of $37^{\text {st }}$ IAS, p. 432-439, San Diego, USA, 1996.

[6] E. G. Marra, J. A. Pomilio. "Self-excited induction generator controlled by a VS-PWM bi-directional converter for rural applications", Proceedings of APEC'98, p. 116122, Anaheim, USA, 1998.

[7] R. Szcesny, M. A. Ronkowski. "New equivalent circuit approach to simulation of converter - induction machine associations", Proceedings of the European Conference on Power Electronics and Applications (EPE'91), p. 4/356-4/361, Firenze, Italy, 1991.

[8] Rodolfo M. M. Martinez. Uma Contribuição ao Estudo e ao Projeto de um Inversor Trifásico de Tensão a Três Niveis, FEEC-UNICAMP - Campinas-SP, Dissertação de MSc., Agosto de 1997.

\section{DADOS BIOGRÁFICOS}

Enes Gonçalves Marra, graduou-se Engenheiro Eletricista e obteve título de Mestre pela Universidade Federal de Uberlândia, Uberlândia (MG), em 1988 e 1993, respectivamente. Entre 1990 e 1991, atuou na área de Proteção de Sistemas Elétricos de Potência na CONPROVE Eng. Ltda. É professor assistente na Escola de Engenharia Elétrica da Universidade Federal de Goiás, Goiânia (GO), desde 1993. Atualmente, é aluno de Doutorado na UNICAMP. É membro da SOBRAEP, do IEEE e da SBA.

José Antenor Pomilio, formou-se em Engenharia Elétrica pela Universidade Estadual de Campinas (UNICAMP), Campinas (SP), em 1983. Obteve os títulos de Mestre e Doutor em Engenharia Elétrica pela mesma universidade em 1986 e 1991, respectivamente. Entre 1988 e 1993 participou do Grupo de Eletrônica de Potência do Laboratório Nacional de Luz Síncroton (CNPq), sendo chefe do Grupo entre 1988 e 1991. Realizou estágio de pós-doutoramento junto ao Departamento de Engenharia Elétrica da Universidade de Pádua, Itália, em 1993/94. É professor do Departamento de Sistemas e Controle de Energia da Faculdade de Engenharia Elétrica e de Computação da UNICAMP. É membro da SOBRAEP, do IEEE e da SBA. 